\title{
Pre- versus postoperative chemoradiotherapy for locally advanced esophageal squamous cell carcinoma
}

\author{
Po-Kuei Hsu, MD, PhD, ${ }^{a}$ Hui-Shan Chen, $\mathrm{PhD},{ }^{\mathrm{b}}$ Chia-Chuan Liu, MD, ${ }^{\mathrm{c}}$ Chien-Sheng Huang, MD, ${ }^{\mathrm{a}}$ \\ Chih-Cheng Hsieh, MD, ${ }^{a}$ Han-Shui Hsu, MD, PhD, ${ }^{a}$ and Shiao-Chi $\mathrm{Wu}, \mathrm{PhD}^{\mathrm{b}}$
}

\begin{abstract}
Objectives: Although preoperative chemoradiation followed by surgery has been recognized as an efficient strategy for esophageal cancer treatments, several studies demonstrate survival benefits of postoperative chemoradiation for those undergoing upfront resection. The optimal sequence of surgery and chemoradiation remains unclear.
\end{abstract}

Methods: Data of 1647 patients with clinical stage II/III esophageal squamous cell carcinoma (ESCC), including 1245 receiving preoperative chemoradiation followed by esophagectomy (pre-OP CRT group) and 402 receiving primary esophagectomy followed postoperative chemoradiation (post-OP CRT group), were obtained from a nationwide database. Propensity score matching identified 286 well-balanced pairs for outcome comparison.

Results: In matched patients, the 3-year overall survival (OS) rates/median survival were not significantly different between the 2 groups $(44.0 \% 3$-year OS/26.0 months; 95\% confidence interval [CI], 18.9-89 38.0 months) in the pre-OP CRT group, versus 37.9\% 3-year OS/23.5 months $(95 \% \mathrm{CI}$, 18.5-29.9 months) in the post-OP CRT group, $P=.3152$ ). The 3 -year disease-free survival rates (DFS)/median survival after surgery were $38.7 \%$ 3-year DFS/16.7 months (95\% CI, 11.9-29.6 months) in the pre-OP CRT group, compared with 30.2\% 3-year DFS/10.4 months (95\% CI, 7.614.0 months $)$ in the post-OP CRT group $(P=.0674)$. In patients who had complete resection, the freedom from recurrence rate at 1 year after surgery was $74.8 \%$ and $67.6 \%$ in pre-OP CRT and post-OP CRT groups, respectively $(P=.2696)$. In the multivariable analysis, treatment modality (pre- or post-OP CRT) was not a significant factor for OS $(P=.258)$ or disease-free survival $(P=.521)$.

Conclusions: Similar outcome can be achieved with postoperative chemoradiotherapy compared with preoperative chemoradiotherapy in patients with locally advanced ESCC. There is little difference between these 2 strategies. (J Thorac Cardiovasc Surg 2017;154:732-40)

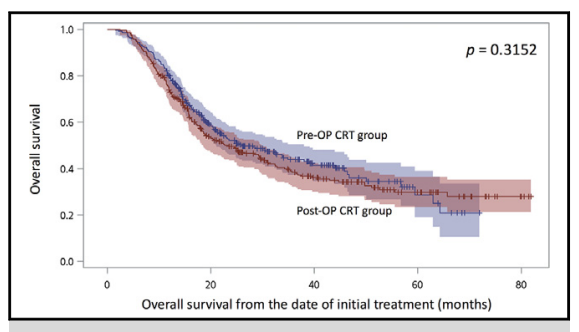

No overall survival difference between pre- and postoperative chemoradiotherapy groups.

\section{Central Message}

No significant difference in overall survival and disease-free survival was observed between pre- and postoperative chemoradiotherapy groups in locally advanced esophageal cancer.

\section{Perspective}

Multimodality therapy is the mainstay of treatments for esophageal cancer, but the optimal sequence is unknown. In 286 well-balanced patients with esophageal squamous cell carcinoma, the overall and disease-free survival rates were not different between pre- and postoperative chemoradiotherapy groups. Our results support the notion that there is little difference between these 2 strategies.

See Editorial Commentary page 741.

See Editorial page 730.

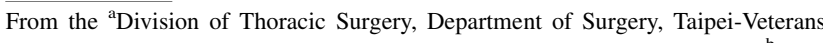
General Hospital and School of Medicine, National Yang-Ming University; ${ }^{\mathrm{b}}$ Institute of Health and Welfare Policy, National Yang-Ming University; and ${ }^{\mathrm{c}}$ Division of Thoracic Surgery, Department of Surgery, Koo Foundation Sun Yat-Sen Cancer Center, Taipei, Taiwan.

P.K.H., H.S.C., and S.C.W. contributed equally to this study.

Received for publication March 23, 2016; revisions received Feb 17, 2017; accepted for publication March 6, 2017; available ahead of print April 27, 2017.

Address for reprints: Po-Kuei Hsu, MD, PhD, Division of Thoracic Surgery, Department of Surgery, Taipei-Veterans General Hospital, No. 201, Sec. 2, Shih-Pai Rd, Taipei, Taiwan (E-mail: hsupokuei@yahoo.com.tw).

0022-5223/\$36.00

Copyright (c) 2017 by The American Association for Thoracic Surgery

http://dx.doi.org/10.1016/j.jtcvs.2017.03.038
}

Esophageal cancer is the seventh most common cause of cancer-related death worldwide. ${ }^{1}$ In 2012, an estimated 455,800 people were diagnosed with esophageal cancer and 400,200 people eventually died due to esophageal

Scanning this $\mathrm{QR}$ code will take you to supplemental figure and tables for this article. 


\section{Abbreviations and Acronyms \\ ESCC = esophageal squamous cell carcinoma \\ Post-OP CRT $=$ postoperative chemoradiation \\ Pre-OP CRT $=$ preoperative chemoradiation}

cancer worldwide. Characterized by dismal prognosis and high frequency of both locoregional and distant disease recurrences, multimodality therapy consisting of chemotherapy, radiotherapy, and surgery has been the mainstay of treatments for esophageal cancer. ${ }^{2-5}$ The strategy of preoperative chemoradiation followed by surgery has been well recognized as an efficient approach. ${ }^{2-4}$ In the Chemoradiotherapy for Oesophageal Cancer Followed by Surgery Study (CROSS) trial, the median overall survival was 49.4 months in the preoperative chemoradiation followed by surgery group versus 24.0 months in the surgery alone group. A significant estimated 5-year overall survival benefit of $13 \%$ ( $47 \%$ vs $34 \%$ ) was noted in favor of the preoperative chemoradiation followed by surgery $\mathrm{arm}^{2}$ On the other hand, several studies have shown survival benefits of postoperative chemoradiation for those who received esophagectomy as initial treatment. ${ }^{5-10}$ For example, our previous study demonstrated that the 3-year overall survival rate was $44.9 \%$ and $28.1 \%$ among patients with esophageal squamous cell carcinoma (ESCC) who underwent esophagectomy with postoperative chemoradiation and those who underwent surgery alone, respectively. ${ }^{5}$

Few studies to date have specifically focused on the prognostic impact of the sequence of surgery and chemoradiation in esophageal cancer, with conflicting results. ${ }^{11,12}$ Hong et $\mathrm{al}^{11}$ studied the Surveillance, Epidemiology, and End Results (SEER) database and demonstrated that preoperative chemoradiation has superior survival over postoperative chemoradiation for locally advanced esophageal cancer. On the other hand, the prospective trial by $\mathrm{Lv}$ et $\mathrm{al}^{12}$ demonstrated that preoperative and postoperative chemoradiation provided similar survival benefit for patients with locally advanced ESCC. As the optimal timing and sequence of surgery and chemoradiation for esophageal cancer remains controversial, this study was conducted to analyze treatment outcomes between patients with ESCC who underwent preoperative chemoradiation followed by surgery and those who had postoperative chemoradiation following primary esophagectomy.

\section{METHODS}

This study's design was reviewed by the institutional review board of the Taipei-Veterans General Hospital, and the requirement for informed consent was waived, due to the retrospective design (VGHIRB No. 2015-06-001BC). Patient data were obtained from Taiwan Cancer Registry database, which is a national population-based database that was implemented in 1979 and is currently administered by the Health Pro- motion Administration, Ministry of Health and Welfare, Executive Yuan, Taiwan. ${ }^{13}$ Hospitals with more than a 50-bed capacity that provide outpatient and hospitalized cancer care are recruited to participate and report all newly diagnosed malignant neoplasms to the registry. The registry currently includes 80 hospitals that account for $>90 \%$ of all cancer cases in Taiwan. Confidentiality was ensured by the Health and Welfare Data Science Center (HWDC), Ministry of Health and Welfare, Executive Yuan, Taiwan. The HWDC encrypts individual identifiers to protect privacy before releasing information to investigators for research purposes.

The inclusion criteria for this study were patients with newly diagnosed clinical stage I, II, and III ESCC between 2008 and 2013, based on their International Classification of Diseases for Oncology (ICD-O3) site codes (C15.0-C15.5, C15.8, and C15.9) and morphology codes (8052, 8070-8074, 8076, 8077, 8083, and 8084); and had undergone preoperative chemoradiation followed by surgical resection (pre-OP CRT group) or surgical resection followed by postoperative chemoradiation (post-OP CRT group) as the initial treatment modality. During the study period, the registry contained data of 11,607 patients with newly diagnosed ESCC. Among them, 1566 and 610 patients with clinical stage I, II, and III ESCC received preoperative and postoperative chemoradiation, respectively, as initial treatments. The exclusion criteria included clinical stage I tumors $(n=284)$, incomplete clinicopathological information $(n=63)$, and loss of follow-up, either in recurrence or survival status $(\mathrm{n}=182)$. After exclusion, the remaining 1647 patients with clinical stage II and III ESCC, including 1245 patients in the pre-OP CRT group and 402 patients in the post-OP CRT group, were considered eligible for this study. In the pre-OP CRT group, the median interval between the start of radiotherapy and surgery was $85(25 \% \mathrm{Q} 1: 72 ; 75 \% \mathrm{Q} 3$ : $105)$ days; whereas the median interval between surgery and the start of radiotherapy was $42(25 \% \mathrm{Q} 1: 34 ; 75 \% \mathrm{Q} 3: 54)$ days in the post-OP CRT group.

Disease recurrence was classified as locoregional failure or distant metastasis. Locoregional failure was defined as recurrence at the anastomotic site, or within the area of the previous resection, and nodal clearance in the cervical, mediastinum, or upper abdomen regions. Distant metastasis included distant lymph node dissemination, hematogenous metastasis to solid organs, or recurrence in the pleura or peritoneal cavities.

Categorical and continuous variables were compared with the $\chi^{2}$ test and Student $t$ test, respectively. Propensity score matching was performed to decrease confounding effects due to nonrandomized assignment. ${ }^{14}$ First, a propensity score for each patient was calculated by logistic regression by using the variables of age, sex, clinical stage, tumor location, differentiation grade, and tumor length. Then a 1:1 matched study group was created with use of a greedy matching algorithm. The missing data were incorporated as a missing value variable without any type of imputation. After matching, 286 well-balanced pairs of patients were identified for outcome comparison. Cox proportional hazards regression model was used for univariable and multivariable survival analyses. Survival curves were plotted using the Kaplan-Meier method and compared with the log-rank test. The overall survival was calculated as the period between the date of initial treatment and the date of death. The disease-free survival was calculated as the interval between the date of surgery and the earliest date of event, which included death, recurrence, and positive surgical margin, because patients with non-R0 resection were considered without a disease-free period. We also calculated the recurrence-free interval (freedom from recurrence), which is the period between the date of complete resection and the date of disease recurrence (but not death). Patients who survived to the end of the follow-up period (December 31, 2014) were censored. All statistical calculations were performed with Statistical Analysis System (version 9.3; SAS Institute, Inc, Cary, NC) and Statistical Product and Service Solutions (version 20; SPSS Inc, Chicago, IL). A $P<.05$ was considered statistically significant. 
TABLE 1. Patient demographics before and after propensity score matching

\begin{tabular}{|c|c|c|c|c|c|c|c|c|c|c|c|c|}
\hline & \multicolumn{4}{|c|}{ Before matching } & \multirow[b]{3}{*}{$P$} & \multirow[b]{3}{*}{ SDif* } & \multicolumn{4}{|c|}{ After matching } & \multirow[b]{3}{*}{$P$} & \multirow[b]{3}{*}{ SDif } \\
\hline & \multicolumn{2}{|c|}{ Pre-OP CRT } & \multicolumn{2}{|c|}{ Post-OP CRT } & & & \multicolumn{2}{|c|}{ Pre-OP CRT } & \multicolumn{2}{|c|}{ Post-OP CRT } & & \\
\hline & $\mathbf{n}$ & $\%$ & $\mathbf{n}$ & $\%$ & & & $\mathbf{n}$ & $\%$ & $\mathbf{n}$ & $\%$ & & \\
\hline Total & 1245 & 100 & 402 & 100 & & & 286 & 100 & 286 & 100 & & \\
\hline Age, years, mean $\pm \mathrm{SD}$ & \multicolumn{2}{|c|}{$54.0 \pm 8.6$} & \multicolumn{2}{|c|}{$54.5 \pm 9.2$} & .351 & 0.053 & \multicolumn{2}{|c|}{$54.4 \pm 8.4$} & \multicolumn{2}{|c|}{$54.6 \pm 9.2$} & .798 & 0.021 \\
\hline Sex & & & & & .795 & & & & & & .404 & \\
\hline Male & 1184 & 95.1 & 381 & 94.8 & & 0.015 & 276 & 96.50 & 272 & 95.10 & & 0.070 \\
\hline Female & 61 & 4.9 & 21 & 5.2 & & 0.015 & 10 & 3.50 & 14 & 4.90 & & 0.070 \\
\hline cStage & & & & & $<.001$ & & & & & & .055 & \\
\hline II & 214 & 17.2 & 203 & 50.5 & & & 92 & 32.17 & 114 & 39.86 & & \\
\hline III & 1031 & 82.8 & 199 & 49.5 & & & 194 & 67.83 & 172 & 60.14 & & \\
\hline cT stage & & & & & $<.001$ & & & & & & 1.000 & \\
\hline $1 / 2$ & 149 & 12.0 & 136 & 33.8 & & 0.539 & 68 & 23.78 & 68 & 23.78 & & 0.000 \\
\hline $3 / 4$ & 1096 & 87.3 & 266 & 66.2 & & 0.539 & 218 & 76.22 & 218 & 76.22 & & 0.000 \\
\hline $\mathrm{cN}$ stage & & & & & $<.001$ & & & & & & .846 & \\
\hline 0 & 155 & 12.4 & 148 & 36.8 & & 0.590 & 69 & 24.13 & 71 & 24.83 & & 0.016 \\
\hline+ & 1090 & 87.6 & 254 & 63.2 & & 0.590 & 217 & 75.87 & 215 & 75.17 & & 0.016 \\
\hline \multicolumn{13}{|l|}{$\mathrm{p}$ (or yp) T stage } \\
\hline T0 & 411 & 33.0 & 0 & 0 & & & 91 & 31.82 & 0 & 0 & & \\
\hline $\mathrm{T} 1$ & 167 & 13.4 & 33 & 8.2 & & & 50 & 17.5 & 21 & 7.34 & & \\
\hline $\mathrm{T} 2$ & 248 & 19.9 & 59 & 14.7 & & & 52 & 18.18 & 38 & 13.29 & & \\
\hline $\mathrm{T} 3$ & 369 & 29.6 & 282 & 70.2 & & & 86 & 30.07 & 205 & 71.68 & & \\
\hline $\mathrm{T} 4$ & 50 & 4.0 & 28 & 7.0 & & & 7 & 2.45 & 22 & 7.69 & & \\
\hline \multicolumn{13}{|l|}{$\mathrm{p}$ (or yp) N stage } \\
\hline No & 868 & 69.7 & 92 & 22.9 & & & 195 & 68.18 & 66 & 23.08 & & \\
\hline N1 & 260 & 20.9 & 166 & 41.3 & & & 66 & 23.08 & 120 & 41.96 & & \\
\hline $\mathrm{N} 2$ & 75 & 6.0 & 112 & 27.9 & & & 16 & 5.59 & 78 & 27.27 & & \\
\hline N3 & 26 & 2.1 & 17 & 4.2 & & & 6 & 2.10 & 12 & 4.20 & & \\
\hline Unknown $\dagger$ & 16 & 1.3 & 15 & 3.7 & & & 3 & 1.05 & 10 & 3.50 & & \\
\hline Tumor length, $\mathrm{cm}$, mean $\pm \mathrm{SD}$ & \multicolumn{2}{|c|}{$5.9 \pm 2.6$} & \multicolumn{2}{|c|}{$4.6 \pm 2.0$} & \multirow[t]{4}{*}{$<.001$} & & \multicolumn{2}{|c|}{$5.2 \pm 2.7$} & & 2.0 & .196 & \\
\hline$\leq 4.6 \ddagger$ & 277 & 22.3 & 233 & 58.0 & & 0.782 & 139 & 48.6 & 145 & 50.7 & & 0.042 \\
\hline$<4.6$ & 637 & 51.2 & 158 & 39.3 & & 0.240 & 147 & 51.4 & 141 & 49.3 & & 0.042 \\
\hline Unknown & 331 & 26.6 & 11 & 2.7 & & & 0 & 0 & 0 & 0 & & \\
\hline Location & & & & & $<.001$ & & & & & & .928 & \\
\hline Upper & 294 & 23.6 & 132 & 32.8 & & 0.151 & 98 & 34.27 & 94 & 32.87 & & 0.035 \\
\hline Middle & 483 & 38.8 & 126 & 31.3 & & 0.157 & 83 & 29.02 & 89 & 31.12 & & 0.046 \\
\hline Lower & 167 & 13.4 & 35 & 8.7 & & 0.206 & 30 & 10.49 & 27 & 9.44 & & 0.030 \\
\hline Unknown & 301 & 24.2 & 109 & 27.1 & & 0.067 & 75 & 26.22 & 76 & 26.57 & & 0.008 \\
\hline Differentiation & & & & & $<.001$ & & & & & & .846 & \\
\hline Good & 27 & 2.2 & 12 & 3.0 & & 0.052 & 8 & 2.80 & 9 & 3.15 & & 0.021 \\
\hline Moderate & 571 & 45.9 & 238 & 59.2 & & 0.270 & 175 & 61.19 & 184 & 64.34 & & 0.065 \\
\hline Poor & 252 & 20.2 & 144 & 35.8 & & 0.352 & 99 & 34.62 & 89 & 31.12 & & 0.074 \\
\hline Unknown & 395 & 31.7 & 8 & 2.0 & & 0.865 & 4 & 1.40 & 4 & 1.40 & & 0.000 \\
\hline Margin status & & & & & $<.001$ & & & & & & $<.001$ & \\
\hline Negative (R0) & 1118 & 89.8 & 311 & 77.4 & & & 257 & 89.86 & 219 & 76.57 & & \\
\hline Positive (R1/2) & 127 & 10.2 & 91 & 22.6 & & & 29 & 10.14 & 67 & 23.43 & & \\
\hline
\end{tabular}

Pre-OP CRT, Preoperative chemoradiotherapy; Post-OP CRT, postoperative chemoradiotherapy; SDif, standardized differences; SD, standard deviation. *SDif values of factors used for propensity score matching are provided. An SDif of $<0.10$ likely denotes a negligible imbalance between case patients and their matched controls. $\dagger$ Definite positive

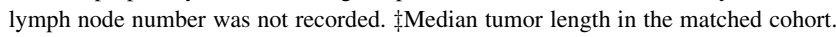

\section{RESULTS}

The clinical and pathological characteristics of the patients before and after propensity score matching are summarized in Table 1. The distribution of propensity scores is displayed in Figure 1. Patients who received preoperative chemoradiation had more advanced tumors in terms of clinical stage III, cT3/4, cN(+), and larger tumor size. With regard to the surgical results, $411(33.3 \%)$ patients in the pre-OP CRT group had no detectable primary tumor and $356(28.6 \%)$ patients achieved complete pathological response (ypT0N0). In the post-OP CRT group, most patients $(n=249,61.9 \%)$ were seen to have pathological 


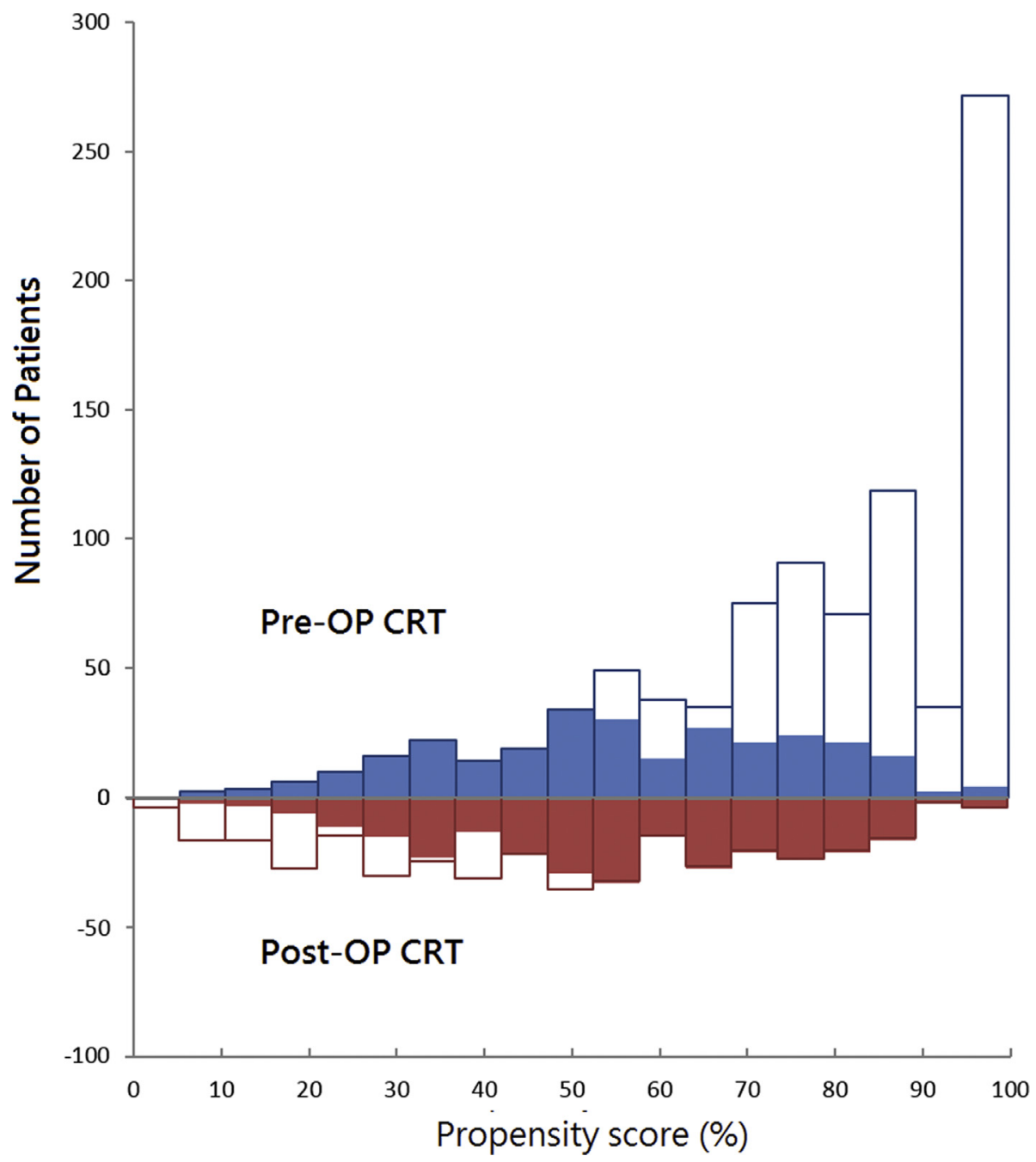

FIGURE 1. Mirrored histogram displaying distribution of propensity scores among matched and unmatched patients. Pre-OP CRT, Preoperative chemoradiotherapy; Post-OP CRT, postoperative chemoradiotherapy.

stage III tumors. In the pre-OP CRT and post-OP-CRT groups, respectively, $10.2 \%$ and $22.6 \%$ of patients had positive margins before matching $(P<.001)$, whereas $10.1 \%$ and $23.4 \%$, respectively, after matching $(P<.001)$, which reflected that positive margin (non-R0 resection) was a common indication for postoperative chemoradiation. Surgical mortality was noted in $27(2.2 \%, 27 / 1245)$ and 0 patients in the pre-OP CRT and post-OP CRT groups, respectively.

In the survival analysis, the median follow-up time was 31.7 months $(95 \%$ confidence interval $[\mathrm{CI}]$, 12.471.9 months) for the surviving patients. In matched patients, the 1-year, 3-year overall survival rates and median survival were $79.3 \%, 44.0 \%$, and 26.0 months $(95 \% \mathrm{CI}, 18.9$ 38.0 months) in the pre-OP CRT group, versus $72.9 \%$, $37.9 \%$, and 23.5 months (95\% CI, 18.5-29.9 months) in the post-OP CRT group $(P=.3152$, Figure 2$)$. The 1-year, 3-year disease-free survival rates and median survival after surgery were $55.7 \%, 38.7 \%$, and 16.7 months (95\% CI, 11.9-29.6 months) in the pre-OP CRT group, compared with $45.1 \%, 30.2 \%$, and 10.4 months (95\% CI, 7.6-14.0 months) in the post-OP CRT group $(P=.0674$, Figure 3$)$. In patients who had complete resection, the freedom from recurrence rate at 1 year after surgery was $74.8 \%$ and $67.6 \%$ in the pre-OP CRT and postOP CRT groups, respectively ( $P=.2696$, Figure 4$)$. There were also no significant differences between the 2 groups in the freedom from locoregional failure $(89.7 \%$ vs $85.2 \%$, $P=.1924)$ and freedom from distant metastasis $(81.3 \%$ vs $77.7 \%, P=.2108$ ) at 1 year (Survival curves including all patients was in Figure E1).

In the Cox regression model based on propensity scorematched patients, only T3/4 stage was an independent prognostic factor for overall survival (Table 2), whereas treatment modality (pre-OP CRT or post-OP CRT) was not $(P=.258$ ). In the subgroup analysis, the significant prognostic factors included ypT3/4 (hazard ratio [HR] 1.84; 95\% CI, 1.33$2.55, P=.0002$ ), and ypN+ (HR 2.07; 95\% CI, 1.50-2.87, $P<.001)$ in the pre-OP CRT group. In the post-OP CRT 


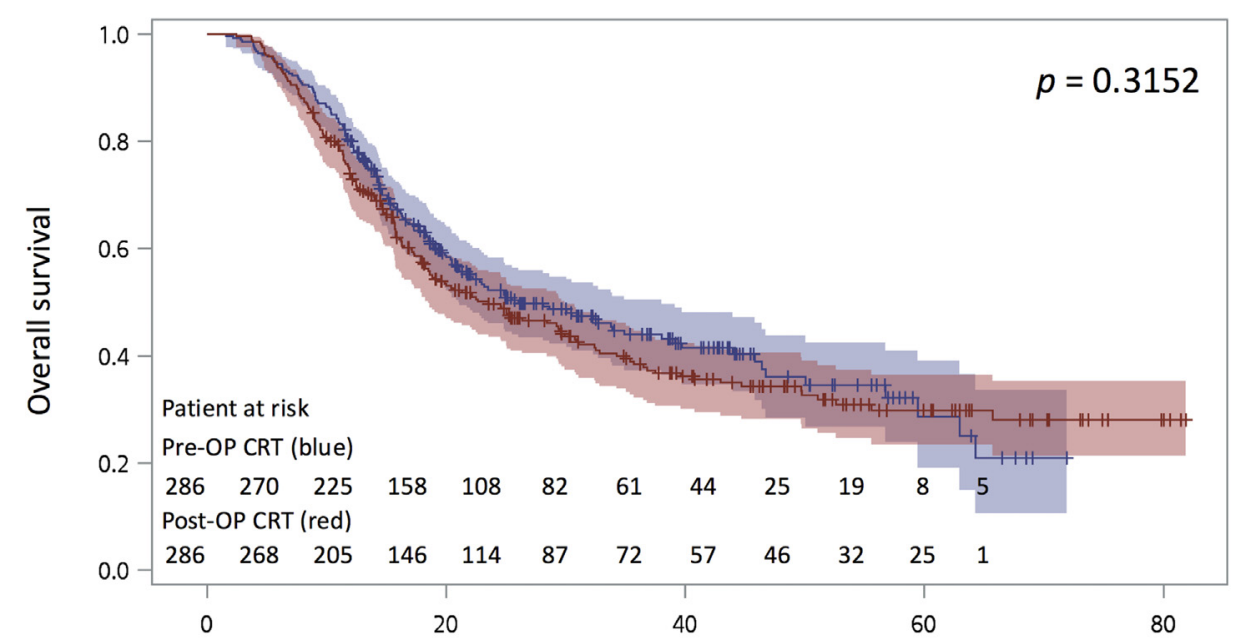

A

Overall survival from the date of initial treatment (months)

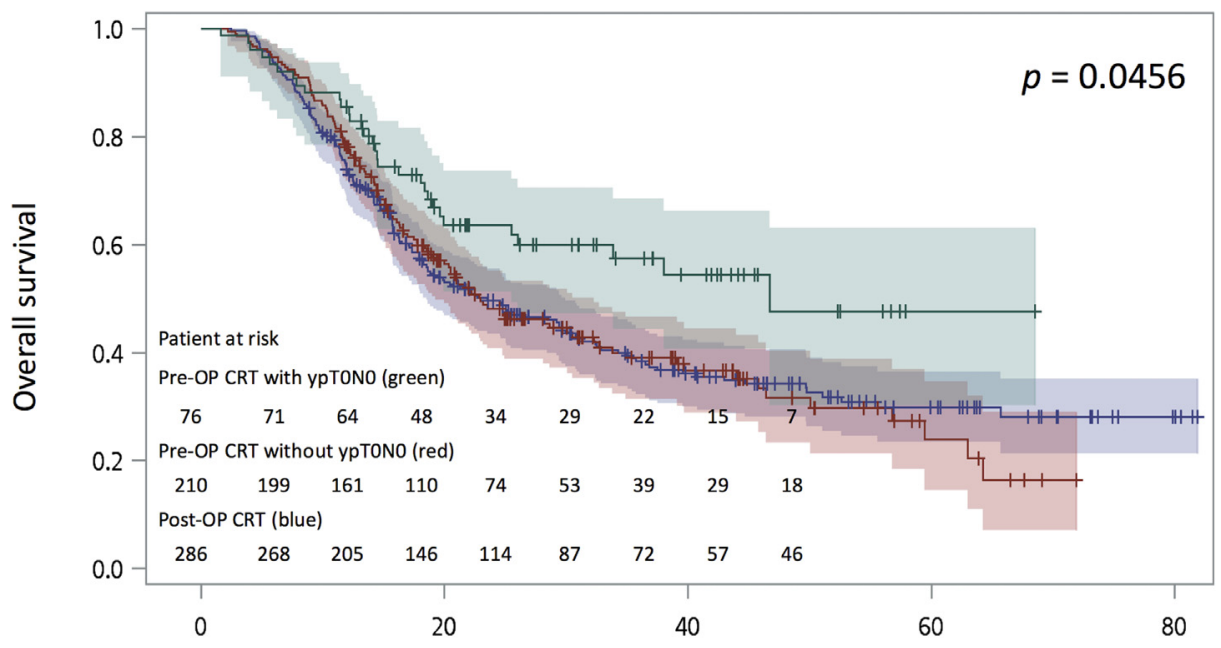

B

Overall survival from the date of initial treatment (months)

FIGURE 2. A, Overall survival, with $95 \%$ confidence interval, of patients in pre-OP CRT (blue line) and post-OP CRT (red line) groups, respectively. B, Overall survival, with $95 \%$ confidence interval, of patients in pre-OP CRT with ypT0N0 (green line) and pre-OP CRT without ypT0N0 (red line), and post-OP CRT (blue line) groups, respectively. Pre-OP CRT, Preoperative chemoradiotherapy; Post-OP CRT, postoperative chemoradiotherapy.

group, the independent prognostic factors included cT3/4 stage (HR 2.06; 95\% CI, 1.36-3.10, $P=.006$ ), pT3/4 stage (HR 1.82; 95\% CI, 1.19-2.79, $P=.006), \mathrm{pN}+($ HR 1.56; $95 \% \mathrm{CI}, 1.06-2.28, P=.023$ ), and tumor length $>4.6 \mathrm{~cm}$ (HR 1.39; 95\% CI, 1.03-1.87, $P=.031$ ). In patients who had complete resection, the only independent prognostic factor for disease-free survival was clinical T stage (cT3/4 vs cT1/2, HR $1.45 ; 95 \%$ CI, 1.06-2.00, $P=.021)$. No difference was observed between pre- and postoperative chemoradiotherapy groups $(P=.521$, Table 3$)$. (Cox regression analysis in all patients were in Tables E1 and E2.)

\section{DISCUSSION}

The dismal prognosis of locally advanced esophageal cancer has motivated multiple strategies in an effort to enhance patient outcome. In this nationwide populationbased study, there was no significant difference in overall survival between pre-OP CRT and post-OP CRT groups. Although the pre-OP CRT group seemed to have better disease-free survival than post-OP CRT group on the Kaplan-Meier plot, this small and nonsignificant difference in survival might be attributable to the proportion of positive margin, which itself was an indication for postoperative chemoradiation and definitively would be higher in the post-OP CRT group.

Preoperative chemoradiation is now widely used because it is believed to be able to eradicate systemic micrometastasis, downstage the tumor, and facilitate the subsequent radical resection, which in turn improves patient survival. The success of the pivotal CROSS trial has established 


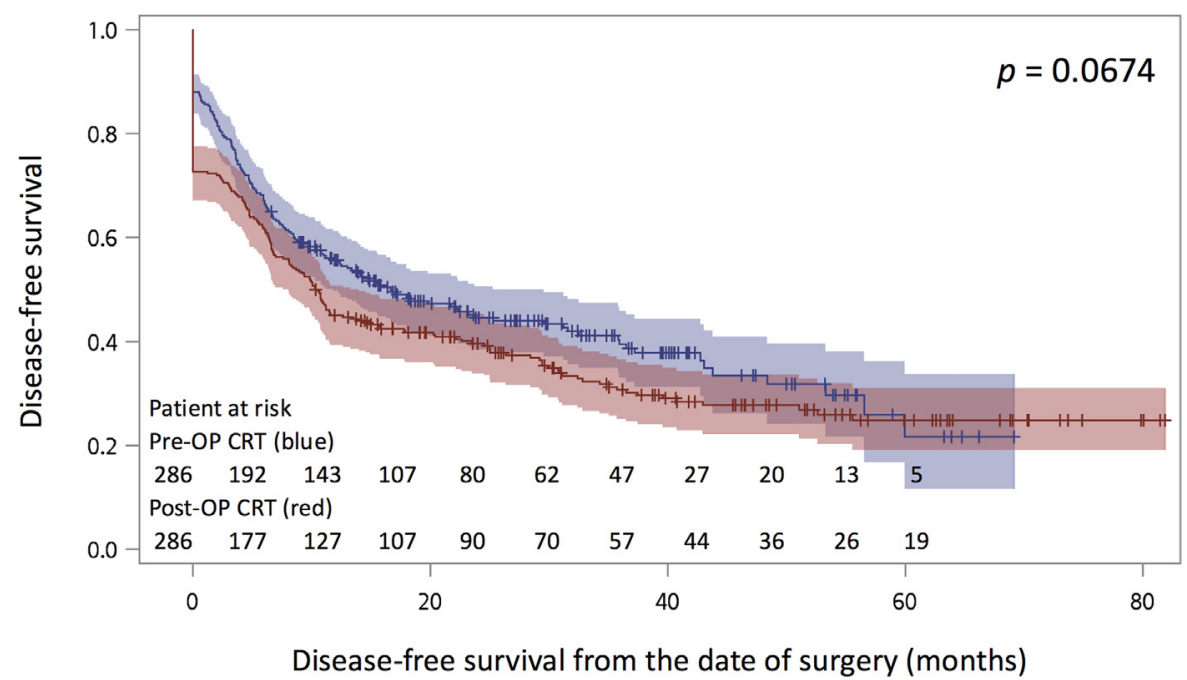

FIGURE 3. Disease-free survival, with $95 \%$ confidence interval, of patients in pre-OP CRT (blue line) and post-OP CRT (red line) groups, respectively. Pre-OP CRT, Preoperative chemoradiotherapy; Post-OP CRT, postoperative chemoradiotherapy.

the role of preoperative chemoradiation followed by esophagectomy for treating locally advanced esophageal cancer. ${ }^{3}$ In the long-term follow-up report, the median overall survivals were 48.6 months (95\% CI, 32.1-65.1 months) after preoperative chemoradiotherapy plus surgery and 24.0 months (95\% CI, 14.2-33.7 months) after surgery alone $(P=.003)$. Compared with patients who underwent surgery alone, patients who underwent preoperative chemoradiotherapy plus surgery experienced significantly less locoregional recurrence ( $22 \%$ vs $38 \%$ ) and distant recurrence $(39 \%$ vs $48 \%))^{4}$

On the other hand, the advantage of postoperative treatments includes the indication for chemoradiation is based on accurate pathological staging, rather than inaccurate clinical staging; thus, the risk of overtreatment would be minimized. Our previous single institute experience has demonstrated that postoperative chemoradiotherapy with a radiation field encompassing the tumor bed as delineated on imaging studies would have better overall survival than surgery alone (3-year overall survival rates: $79.0 \%$ vs $57.0 \%, \quad P=.016){ }^{6}$ The subsequent nationwide population-based study also showed that esophagectomy with postoperative chemoradiation is significantly more effective than surgery alone for increasing overall survival among patients with ESCC. ${ }^{5}$ The median survival and 3year survival rates were 27.2 months $(95 \%$ CI, 17.640.3 months) and $44.9 \%$ among patients who underwent esophagectomy with postoperative chemoradiation,

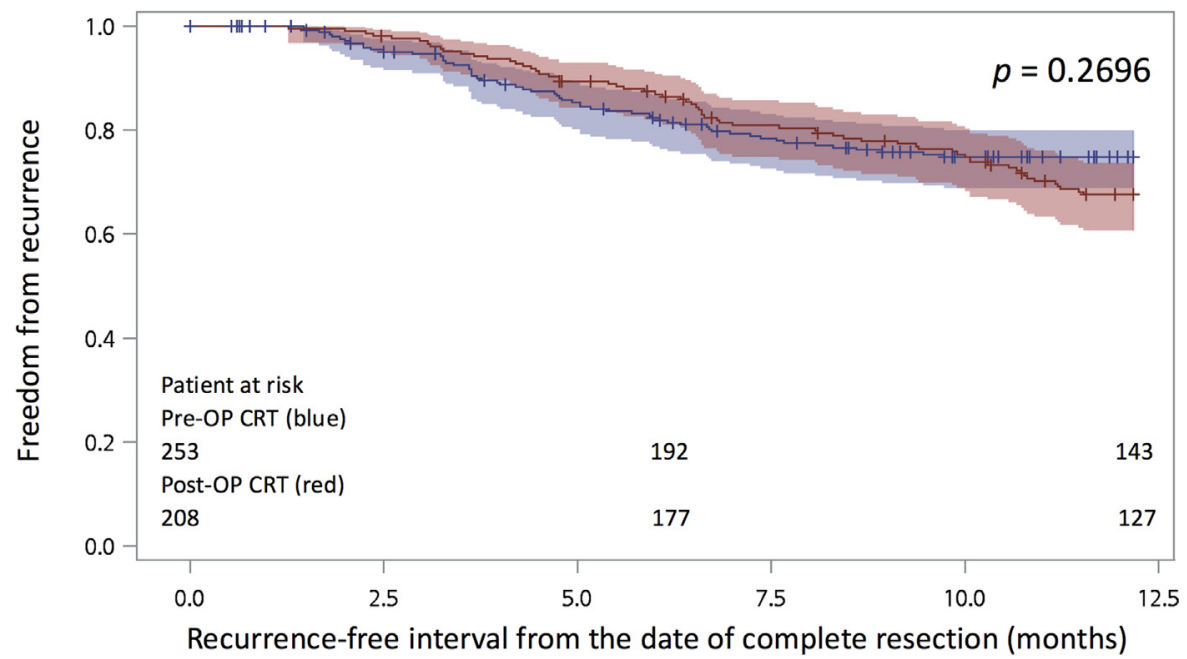

FIGURE 4. Recurrence-free interval, with $95 \%$ confidence interval, of patients in pre-OPCRT (blue line) and post-OP CRT (red line) groups, respectively, at 1 year after surgery. Pre-OP CRT, Preoperative chemoradiotherapy; Post-OP CRT, postoperative chemoradiotherapy. 
TABLE 2. Cox regression analysis in matched groups for overall survival

\begin{tabular}{|c|c|c|c|c|}
\hline & \multicolumn{2}{|c|}{ Univariable analysis } & \multicolumn{2}{|c|}{ Multivariable analysis } \\
\hline & HR $(95 \%$ CI $)$ & $\boldsymbol{P}$ & HR $(95 \%$ CI) & $P$ \\
\hline \multicolumn{5}{|l|}{ Age, y } \\
\hline$\leq 55$ & 1 & & 1 & \\
\hline$>55$ & $1.02(0.82-1.27)$ & .863 & $1.00(0.80-1.25)$ & .992 \\
\hline \multicolumn{5}{|l|}{ Sex } \\
\hline Male & 1 & & 1 & \\
\hline Female & $0.71(0.38-1.32)$ & .276 & $0.79(0.42-1.49)$ & .467 \\
\hline \multicolumn{5}{|l|}{ cT stage } \\
\hline $\mathrm{T} 1 / 2$ & 1 & & 1 & \\
\hline $\mathrm{T} 3 / 4$ & $1.53(1.16-2.03)$ & .003 & $1.47(1.10-1.96)$ & .010 \\
\hline \multicolumn{5}{|l|}{ cN stage } \\
\hline No & 1 & & 1 & \\
\hline $\mathrm{N}+$ & $1.01(0.78-1.30)$ & .957 & $1.02(0.78-1.33)$ & .885 \\
\hline \multicolumn{5}{|l|}{ Location } \\
\hline Lower third & 1 & & 1 & \\
\hline Middle third & $1.09(0.83-1.44)$ & .548 & $1.08(0.82-1.43)$ & .592 \\
\hline Upper third & $1.15(0.77-1.72)$ & .490 & $1.07(0.71-1.63)$ & .739 \\
\hline \multicolumn{5}{|l|}{ Differentiation } \\
\hline Good & 1 & & 1 & \\
\hline Moderate & $0.63(0.36-1.10)$ & .101 & $0.66(0.37-1.16)$ & .149 \\
\hline Poor & $0.86(0.49-1.53)$ & .606 & $0.89(0.50-1.59)$ & .687 \\
\hline \multicolumn{5}{|l|}{ Tumor length, $\mathrm{cm}$} \\
\hline$\leq 4.6$ & 1 & & 1 & \\
\hline$>4.6$ & $1.11(0.90-1.39)$ & .329 & $1.07(0.86-1.34)$ & .540 \\
\hline \multicolumn{5}{|c|}{ Treatment modality } \\
\hline Pre-OP CRT & 1 & & 1 & \\
\hline Post-OP CRT & $1.12(0.90-1.39)$ & .316 & $1.14(0.91-1.41)$ & .258 \\
\hline
\end{tabular}

$H R$, Hazard ratio; $C I$, confidence interval; Pre-OP CRT, preoperative chemoradiotherapy; Post-OP CRT, postoperative chemoradiotherapy.

compared with 18.2 months (95\% CI, 14.3-24.5 months) and $28.1 \%$ among patients who underwent surgery alone $(P=.0055)$. In the literature, several studies also suggested postoperative chemoradiation may be beneficial in patients with esophageal cancer with certain characteristics, such as positive nodal involvement and extracapsular invasion. ${ }^{7-10}$ The toxicity associated with chemoradiation is always a concern for patients after esophagectomy. Before the advent of minimally invasive esophagectomy, patients who undergo esophagectomy are typically debilitated and unable to withstand any postoperative treatments. With the advancement of endoscopic surgical approach, the early restoration of physical function may render patients better able to tolerate adjuvant therapy. ${ }^{15}$ According to our previous report and the phase II study by the Cleveland Clinic group, postoperative 5-fluorouracil and cisplatinbased chemoradiation has acceptable toxicity for patients with esophageal cancer after primary surgical resection. ${ }^{6,16}$ Taken together with other reports, the results from current study indeed provide a basis to reappraise the role of postoperative chemoradiation in ESCC.

To determine the optimal timing and sequence of surgery and chemoradiation for esophageal cancer, Hong et $\mathrm{al}^{11}$
TABLE 3. Cox regression analysis for disease-free survival in matched patients who had curative resection

\begin{tabular}{|c|c|c|c|c|}
\hline & \multicolumn{2}{|c|}{ Univariable analysis } & \multicolumn{2}{|c|}{ Multivariable analysis } \\
\hline & HR $(95 \%$ CI $)$ & $\boldsymbol{P}$ & HR $(95 \%$ CI $)$ & $P$ \\
\hline \multicolumn{5}{|l|}{ Age, y } \\
\hline$\leq 55$ & 1 & & 1 & \\
\hline$>55$ & $1.08(0.85-1.39)$ & .522 & $1.04(0.81-1.33)$ & .763 \\
\hline \multicolumn{5}{|l|}{ Sex } \\
\hline Male & 1 & & 1 & \\
\hline Female & $0.53(0.24-1.19)$ & .125 & $0.55(0.24-1.24)$ & .149 \\
\hline \multicolumn{5}{|l|}{ cT stage } \\
\hline $\mathrm{T} 1 / 2$ & 1 & & 1 & \\
\hline $\mathrm{T} 3 / 4$ & $1.52(1.13-2.06)$ & .007 & $1.45(1.06-2.00)$ & .021 \\
\hline \multicolumn{5}{|l|}{$\mathrm{cN}$ stage } \\
\hline No & 1 & & 1 & \\
\hline $\mathrm{N}+$ & $0.91(0.68-1.21)$ & .498 & $0.89(0.66-1.20)$ & .428 \\
\hline \multicolumn{5}{|l|}{ Location } \\
\hline Lower third & 1 & & 1 & \\
\hline Middle third & $0.93(0.68-1.27)$ & .662 & $0.89(0.65-1.23)$ & .484 \\
\hline Upper third & $1.09(0.67-1.77)$ & .726 & $0.96(0.59-1.58)$ & .882 \\
\hline \multicolumn{5}{|l|}{ Differentiation } \\
\hline Good & 1 & & 1 & \\
\hline Moderate & $0.65(0.33-1.28)$ & .211 & $0.69(0.35-1.35)$ & .279 \\
\hline Poor & $0.92(0.46-1.83)$ & .814 & $0.97(0.49-1.95)$ & .940 \\
\hline \multicolumn{5}{|l|}{ Tumor length, $\mathrm{cm}$} \\
\hline$\leq 4.6$ & 1 & & 1 & \\
\hline$>4.6$ & $1.06(0.83-1.35)$ & .656 & $0.98(0.76-1.26)$ & .885 \\
\hline \multicolumn{5}{|c|}{ Treatment modality } \\
\hline Pre-OP CRT & 1 & & 1 & \\
\hline Post-OP CRT & $1.06(0.82-1.35)$ & .673 & $1.09(0.85-1.39)$ & .521 \\
\hline
\end{tabular}

compared the outcome of pre- and postoperative chemoradiotherapy in nonmetastatic $\mathrm{T} 3+$ or $\mathrm{N}+$ esophageal cancer by using the SEER-Medicare database. The multivariable analysis controlling for age, marital status, and histology showed that preoperative chemoradiotherapy had better overall survival versus postoperative chemoradiotherapy (HR 1.63; $P=.03$ ). On the other hand, the prospective study by Lv et al ${ }^{12}$ showed conflicting results. The 5-year overall survival rates were $43.5 \%$ and $42.3 \%$ in pre- and postoperative chemoradiotherapy groups, respectively, in patients with clinical stage II and III ESCC. The 5-year progression-free survival rates were $37.5 \%$ and $37.2 \%$ in pre- and postoperative chemoradiotherapy groups, respectively, which indicated no significant survival differences between the pre- and postoperative chemoradiotherapy groups. Similar concepts also have been observed in esophageal adenocarcinoma. Zahoor et al ${ }^{17}$ compared survival for patients with stage II or higher esophageal adenocarcinoma treated with primary esophagectomy followed by postoperative therapy or preoperative therapy protocol. In propensity-matched pairs, the HR for death and recurrence patterns were similar between the 2 groups, which 
suggested that the strategy of primary esophagectomy followed by postoperative chemotherapy does not negatively influence survival compared with the preoperative therapy protocol.

The rate of complete pathologic response in the preoperative chemoradiotherapy group was $28.6 \%$, which is compatible with other values reported in the literature. ${ }^{18,19}$ But in the CROSS trial, the rate was significantly higher in squamous cell carcinoma (49\%) than in adenocarcinoma $(23 \%)$, leading to an impression that squamous cell carcinoma is more responsive to chemoradiation. In fact, a recent systematic review also suggested preoperative chemoradiotherapy with paclitaxel plus platinum regimen to be a better treatment than platinum plus 5-fluorouracil regimen for locoregional ESCC. ${ }^{20}$ Moreover, the role of surgery in patients with squamous cell carcinoma who achieve complete clinical response after chemoradiation is still under debate. If more systematic planned resections were performed (eg, in a prospective randomized trial), the pathologic complete response rate would be higher; in contrast, if a more nonoperative approach with intensive surveillance (eg, symptom relief after preoperative chemoradiation) were chosen, the rate would be lower.

Although this current study attempted to find balanced groups of patients with a propensity score-matching method, there might still be hidden unmeasurable factors that influenced treatment options and survival. For example, surgeons may perform primary esophagectomy for patients with clinically N1, but with limited lymph node involvement, whereas they will suggest preoperative chemoradiation for those with bulky clinical N1 lymph nodes. In patients receiving preoperative chemoradiation, surgeons might refuse surgery for patients with comorbidities or poor clinical response, selecting only patients assumed to have better outcome for surgical resection. Similarly, in patients after primary esophagectomy, postoperative chemoradiation was performed only for those considered to have ominous prognostic factors based on pathologic examination. The impact of these and other possible sources of bias could not be precisely evaluated. In addition, we did not include patients who failed to survive preoperative chemoradiotherapy or those who failed to survive primary surgery and could not complete treatments, which violates the intention-to-treat principle. Furthermore, because the Taiwan Cancer Registry database is a prospectively maintained database, it has limitations that are inherent to all retrospective studies, such as lacking detailed information on staging workup, chemoradiation regimens, radiation fields, surgical techniques, surgical complications, and follow-up protocol. Furthermore, in patients who received preoperative chemoradiation and esophagectomy in different hospitals, some pretreatment factors (eg, tumor length, location, and differentiation) could not be precisely recorded. However, as positron emission tomography/computed tomography (PET/CT) is reimbursed by the Taiwan National Health Insurance system, almost all patients with a diagnosis of esophageal cancer receive PET/CT for free as a staging workup. Moreover, the only regimen reimbursed by Taiwan National Insurance System is cisplatin/5-fluorouracil-based chemotherapy, which is frequently used preoperatively or postoperatively. In addition, the high incidence of upper/middle-third tumors in Taiwan has made McKweon esophagectomy, which means esophagogastric anastomosis at neck, a routine procedure. ${ }^{21}$ The Ivor-Lewis and transhiatal esophagectomy, which are frequently used for adenocarcinoma of lower-third esophagus or esophagogastric junction, are rarely performed for ESCC. This study is also limited by the absence of documentation of the patient's comorbidities, which are often used in propensity matching in retrospective studies, especially when they play a pivotal role in deciding to use additional treatments or not. However, all patients in the current study underwent trimodality treatments, only the sequence was different, which means that all included patients were considered to be able to complete these treatments. In Taiwan, there is increasing adoption of preoperative chemoradiotherapy protocol, but still some prefer upfront surgery. Therefore, the treatment protocol decision mostly depends on the surgeon's or institution's preferences. Because patients' comorbidities were not a factor in deciding which treatment protocol they received, we did not include them during the propensity matching.

In conclusion, given the limitations of the database and our study design, we could not show any difference in overall survival or disease-free survival between preoperative and postoperative chemoradiotherapy groups in clinical stage II and III ESCC. Although preoperative chemoradiation followed by surgery is the more frequently chosen approach for locally advanced esophageal cancer, our results suggested that there is not a substantial difference between the preoperative and postoperative chemoradiotherapy strategies.

\section{Conflict of Interest Statement}

Authors have nothing to disclose with regard to commercial support.

\section{References}

1. Torre LA, Bray F, Siegel RL, Ferlay J, Lortet-Tieulent J, Jemal A. Global cancer statistics, 2012. CA Cancer J Clin. 2015;65:87-108.

2. Jang R, Darling G, Wong RK. Multimodality approaches for the curative treatment of esophageal cancer. J Natl Compr Canc Netw. 2015;13:229-38.

3. Van Hagen P, Hulshof MC, van Lanschot JJ, Steyerberg EW, van Berge Henegouwen MI, Wijnhoven BP, et al. Preoperative chemoradiotherapy for esophageal or junctional Cancer. N Engl J Med. 2012;366:2074-84.

4. Shapiro J, van Lanschot JJ, Hulshof MC, van Hagen P, van Berge Henegouwen MI, Wijnhoven BP, et al. Neoadjuvant chemoradiotherapy plus surgery versus surgery alone for oesophageal or junctional cancer (CROSS): longterm results of a randomised controlled trial. Lancet Oncol. 2015;16:1090-8.

5. Hwang JY, Chen HS, Hsu PK, Chao YK, Wang BY, Huang CS, et al. A propensity-matched analysis comparing survival after esophagectomy followed 
by adjuvant chemoradiation to surgery alone for esophageal squamous cell carcinoma. Ann Surg. 2016;264:100-6.

6. Hsu PK, Huang CS, Wang BY, Wu YC, Hsu WH. Survival benefits of postoperative chemoradiation in lymph node-positive esophageal squamous cell carcinoma. Ann Thorac Surg. 2014;97:1734-41.

7. Bedard ELR, Inculet RI, Malthaner RA, Brecevic E, Vincent M, Dar R. The role of surgery and postoperative chemoradiation therapy in patients with lymph node positive esophageal carcinoma. Cancer. 2001;91:2423-30.

8. Rice TW, Adelstein DJ, Chidel MA, Rybicki LA, DeCamp MM, Murthy SC, et al. Benefit of postoperative adjuvant chemoradiotherapy in locoregionally advanced esophageal carcinoma. J Thorac Cardiovasc Surg. 2003;126:1590-6.

9. Chen J, Pan J, Liu J, Li J, Zhu K, Zheng X, et al. Postoperative radiation therapy with or without concurrent chemotherapy for node-positive thoracic esophageal squamous cell carcinoma. Int J Radiat Oncol Biol Phys. 2013;86:671-7.

10. Wang ZW, Luan ZP, Zhang W, Dong W, Fu CR, Wang YN, et al. Postoperative chemoradiotherapy improves survival in esophageal squamous cell cancer with extracapsular lymph node extension. Neoplasma. 2014;61:732-8.

11. Hong JC, Murphy JD, Wang SJ, Koong AC, Chang DT. Chemoradiotherapy before and after surgery for locally advanced esophageal cancer: a SEERMedicare analysis. Ann Surg Oncol. 2013;20:3999-4007.

12. Lv J, Cao XF, Zhu B, Tao L, Wang DD. Long-term efficacy of perioperative chemoradiotherapy on esophageal squamous cell carcinoma. World J Gastroenterol. 2010;16:1649-54.

13. Chiang CJ, You SL, Chen CJ, Yang YW, Lo WC, Lai MS. Quality assessment and improvement of nationwide cancer registration system in Taiwan: a review. Jpn J Clin Oncol. 2015;45:291-6.

14. Winger DG, Nason KS. Propensity-score analysis in thoracic surgery: when, why, and an introduction to how. J Thorac Cardiovasc Surg. 2016;151:1484-7.
15. Wang H, Shen Y, Feng M, Zhang Y, Jiang W, Xu S, et al. Outcomes, quality of life, and survival after esophagectomy for squamous cell carcinoma: a propensity score-matched comparison of operative approaches. J Thorac Cardiovasc Surg. 2015; 149:1006-14.

16. Adelstein DJ, Rice TW, Rybicki LA, Saxton JP, Videtic GMM, Murphy SC, et al. Mature results from a phase II trial of postoperative concurrent chemoradiotherapy for poor prognosis cancer of the esophagus and gastroesophageal junction. $J$ Thorac Oncol. 2009;4:1264-9.

17. Zahoor H, Luketich JD, Levy RM, Awais O, Winger DG, Gibson ML, et al. A propensity-matched analysis comparing survival after primary minimally invasive esophagectomy followed by adjuvant therapy to neoadjuvant therapy for esophagogastric adenocarcinoma. J Thorac Cardiovasc Surg. 2015;149:538-47.

18. Meguid RA, Hooker CM, Taylor JT, Kleinberg LR, Cattaneo SM, Sussman MS, et al. Recurrence after neoadjuvant chemoradiation and surgery for esophageal cancer: does the pattern of recurrence differ for patients with complete response and those with partial or no response? J Thorac Cardiovasc Surg. 2009;138:1309-17.

19. Donahue JM, Nichols FC, Li Z, Schomas DA, Allen MS, Cassivi SD, et al. Complete pathologic response after neoadjuvant chemoradiotherapy for esophageal cancer is associated with enhanced survival. Ann Thorac Surg. 2009;87:392-8.

20. Huang TC, Hsu CH, Lin CC, Tu YK. Systematic review and network metaanalysis: neoadjuvant chemoradiotherapy for locoregional esophageal cancer. Jpn J Clin Oncol. 2015;45:1023-8.

21. Perry Y, Fernando HC. Three-field minimally invasive esophagectomy: current results and technique. J Thorac Cardiovasc Surg. 2012;144:S63-6.

Key Words: adjuvant, chemoradiotherapy, esophageal cancer, neoadjuvant 


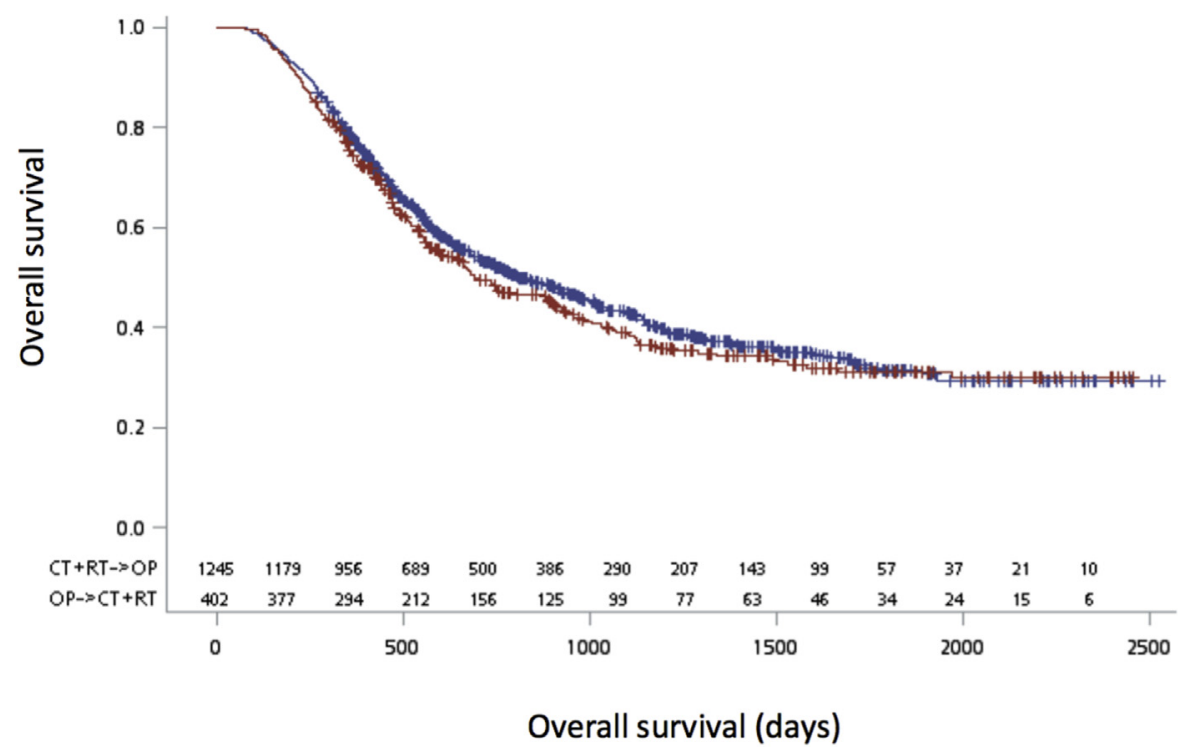

FIGURE E1. Survival curves including all patients (blue: pre-OP CRT group; red: post-OP CRT group, $P=.264$ ).

TABLE E1. Cox regression analysis in all patients for overall survival

\begin{tabular}{|c|c|c|}
\hline & \multicolumn{2}{|c|}{ Multivariable analysis } \\
\hline & HR $(95 \%$ CI $)$ & $P$ \\
\hline \multicolumn{3}{|l|}{ Age, y } \\
\hline$\leq 55$ & 1 & \\
\hline$>55$ & $1.12(0.97-1.29)$ & .114 \\
\hline \multicolumn{3}{|l|}{ Sex } \\
\hline Male & 1 & \\
\hline Female & $0.87(0.62-1.22)$ & .425 \\
\hline \multicolumn{3}{|l|}{ cT stage } \\
\hline $\mathrm{T} 1 / 2$ & 1 & \\
\hline $\mathrm{T} 3 / 4$ & $1.20(0.97-1.48)$ & .088 \\
\hline \multicolumn{3}{|l|}{$\mathrm{cN}$ stage } \\
\hline No & 1 & \\
\hline $\mathrm{N}+$ & $1.09(0.90-1.32)$ & .379 \\
\hline \multicolumn{3}{|l|}{ Location } \\
\hline Lower third & 1 & \\
\hline Middle third & $1.10(0.91-1.33)$ & .316 \\
\hline Upper third & $1.44(1.12-1.84)$ & .004 \\
\hline \multicolumn{3}{|l|}{ Differentiation } \\
\hline Good & 1 & \\
\hline Moderate & $0.71(0.48-1.07)$ & .104 \\
\hline Poor & $0.94(0.62-1.43)$ & .777 \\
\hline \multicolumn{3}{|l|}{ Tumor length, cm } \\
\hline$\leq 4.6$ & 1 & \\
\hline$>4.6$ & $1.09(0.92-1.28)$ & .315 \\
\hline \multicolumn{3}{|c|}{ Treatment modality } \\
\hline Pre-OP CRT & 1 & \\
\hline Post-OP CRT & $1.16(0.97-1.39)$ & .110 \\
\hline
\end{tabular}


TABLE E2. Cox regression analysis for disease-free survival in all patients who had curative resection

\begin{tabular}{|c|c|c|}
\hline & \multicolumn{2}{|c|}{ Multivariable analysis } \\
\hline & HR $(95 \%$ CI $)$ & $P$ \\
\hline \multicolumn{3}{|l|}{ Age, y } \\
\hline$\leq 55$ & 1 & \\
\hline$>55$ & $1.17(0.99-1.37)$ & .063 \\
\hline \multicolumn{3}{|l|}{ Sex } \\
\hline Male & 1 & \\
\hline Female & $0.91(0.63-1.31)$ & .605 \\
\hline \multicolumn{3}{|l|}{ cT stage } \\
\hline $\mathrm{T} 1 / 2$ & 1 & \\
\hline $\mathrm{T} 3 / 4$ & $1.12(0.89-1.41)$ & .333 \\
\hline \multicolumn{3}{|l|}{$\mathrm{cN}$ stage } \\
\hline No & 1 & \\
\hline $\mathrm{N}+$ & $1.02(0.82-1.27)$ & .844 \\
\hline \multicolumn{3}{|l|}{ Location } \\
\hline Lower third & 1 & \\
\hline Middle third & $0.94(0.76-1.15)$ & .520 \\
\hline Upper third & $1.30(0.98-1.72)$ & .066 \\
\hline \multicolumn{3}{|l|}{ Differentiation } \\
\hline Good & 1 & \\
\hline Moderate & $0.58(0.37-0.90)$ & .016 \\
\hline Poor & $0.78(0.49-1.23)$ & .280 \\
\hline \multicolumn{3}{|l|}{ Tumor length, $\mathrm{cm}$} \\
\hline$\leq 4.6$ & 1 & \\
\hline$>4.6$ & $1.12(0.94-1.34)$ & .215 \\
\hline \multicolumn{3}{|c|}{ Treatment modality } \\
\hline Pre-OP CRT & 1 & \\
\hline Post-OP CRT & $1.11(0.90-1.37)$ & .336 \\
\hline
\end{tabular}

